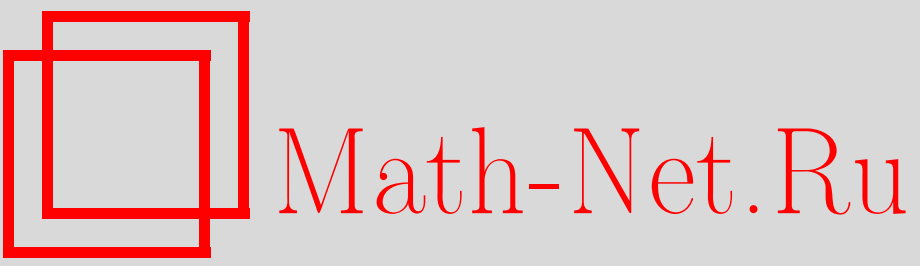

В. Ю. Глотов, В. М. Головизнин, Б. Н. Четверушкин, Баланснохарактеристические разностные схемы для уравнений параболического типа, Матем. моделирование, 2020, том 32, номер 4, 94-106

DOI: https://doi.org/10.20948/mm-2020-04-07

Использование Общероссийского математического портала Math-Net.Ru подразумевает, что вы прочитали и согласны с пользовательским соглашением

http://www.mathnet.ru/rus/agreement

Параметры загрузки:

IP: 54.164 .48 .24

26 апреля 2023 г., 14:12:46 


\title{
БАЛАНСНО-ХАРАКТЕРИСТИЧЕСКИЕ РАЗНОСТНЫЕ СХЕМЫ ДЛЯ УРАВНЕНИЙ ПАРАБОЛИЧЕСКОГО ТИПА
}

\author{
(C) 2020 2. $\quad$ В.Ю. Глотов ${ }^{1}$, В.М. Головизнин ${ }^{2}$, Б.Н. Четверуикин \\ ${ }^{1}$ Институт проблем безопасного развития атомной энергетики (ИБРАЭ) РАН \\ ${ }^{2}$ МГУ имени М.В. Ломоносова \\ ${ }^{3}$ Институт прикладной математики им. М.В. Келдыша РАН \\ glotov-v@ya.ru; gol@ibrae.ac.ru
}

Работа выполнена при поддержке РНФ (грант 19-11-00104).

DOI: $10.20948 / \mathrm{mm}-2020-04-07$

Предложен новый численный алгоритм для решений параболических уравнений по балансно-характеристическим разностным схемам, объединяющим достоинства консервативных и характеристических разностных схем. Основным достоинством нового алгоритма является то, что он является явным и реализуется на максимально компактном вычислительном шаблоне в одну пространственно-временную расчетную ячейку. Он идеально масштабируется и оказывается чрезвычайно экономичным в программных комплексах, реализующих схему КАБАРЕ, поскольку использует уже существующую там структуру данных.

Ключевые слова: уравнения параболического типа, балансно-характеристические разностные схемы, гиперболизация, схема КАБАРЕ.

\section{BALANCE\&CHARACTERISTIC FINITE DIFFERENCE SCHEMES FOR THE EQUATIONS OF THE PARABOLIC TYPE}

\author{
V.Y. Glotov ${ }^{1}$, V.M. Goloviznin', B.N. Chetverushkin ${ }^{3}$ \\ ${ }^{1}$ IBRAE RAS \\ ${ }^{2}$ Lomonosov Moscow State University \\ ${ }^{3}$ Keldysh Institute of Applied Mathematics RAS
}

A new numerical algorithm for solving parabolic equations by means of balance\&characteristic difference schemes is proposed. The balance\&characteristic schemes are combining the advantages of conservative and characteristic schemes. The main advantage of the new algorithm is that it is explicit and is implemented on the most compact computational template in one space-time computational cell. It scales perfectly and is extremely economical in software systems that implement the CABARET scheme because it uses the existing data structure there.

Keywords: parabolic type equations, balance\&characteristic difference schemes, hyperbolization, CABARET scheme. 


\section{Введение}

При прямом численном моделировании турбулентных течений сеточными методами, как правило, используются явные аппроксимации конвективных потоков, приводящие к ограничению допустимого шага интегрирования по времени. В соответствии с критерием Куранта-Фридрихса-Леви, для вычислительной устойчивости явных алгоритмов сеточного переноса необходимо, чтобы за один временной шаг $\tau$ возмущение, имеющее характерную скорость $c$, распространялось на расстояние, не превышающее характерных размеров ячейки расчетной сетки $h$.

Ограничение $\tau \leq h / c$, естественное для систем уравнений гиперболического типа, при явной аппроксимации компонент тензора вязких напряжений, дополняется ограничением $\tau \leq h^{2} / 2 v$ ( $v-$ коэффициент молекулярной вязкости), характерным для явных разностных схем параболического типа. При больших числах Рейнольдса $\operatorname{Re} \geq 10^{3}$ ( $\operatorname{Re}=c L / v, L$ - характерный размер области) и расчетных сетках, типичных для низкорейнольдсовских расчетов $\left(y^{+} \sim 1\right)$, последнее не проявляется, что дает возможность использовать в расчетах «дешевую» явную аппроксимацию сил вязкости [1-3]. Нарушение условий параболической устойчивости происходит только на очень подробных сетках, когда $y^{+} \ll 1$.

В задачах турбулентного тепломассопереноса (например, в ядерных реакторах) наряду с уравнениями Навье-Стокса приходится решать и уравнение теплопроводности (конвекции - диффузии) с доминирующей конвекцией. Для молекулярных сред диффузия тепла и импульса происходит по одним и тем же законам, поэтому отношение коэффициента вязкости $v$ к коэффициенту теплопроводности $\sigma$ (число Прандтля) имеет величину порядка единицы: $(\operatorname{Pr}=v / \sigma \sim 1)$. Это позволяет также использовать явную аппроксимацию диффузионных членов $[1,3]$.

Ситуация существенно меняется в случае жидкометаллических теплоносителей, электронная теплопроводность которых в сотни раз превосходит молекулярную $\left(\operatorname{Pr} \sim 10^{-2}-10^{-3}\right)$. Явная аппроксимация диффузионных членов приводит в этом случае к сильному измельчению временного шага и существенному увеличению продолжительности расчетов. Кроме того, при $y^{+} \ll 1$ начинает доминировать квадратичное ограничение. Переход к неявной аппроксимации усложняет вычислительный алгоритм и также удлиняет расчеты. Хотелось бы модифицировать явные вычислительные алгоритмы для уравнений параболического типа таким образом, чтобы область их вы- 
числительной устойчивости слабо зависела от коэффициента теплопроводности и имела асимптотику типа $\tau \leq h^{\gamma}, \gamma \in(1,2]$.

Целью настоящей работы является разработка явного вычислительного алгоритма для уравнений параболического типа с областью устойчивости, не зависящей от коэффициента теплопроводности, и зависимостью $\tau \leq h^{\gamma}$, $\gamma \in(1,2]$, а также получение достаточных условий его устойчивости.

Гиперболизация уравнений параболического типа. Без ограничения общности и для простоты изложения все математические выкладки в этой статье будем проводить для уравнения теплопроводности.

Уравнение теплопроводности представляет собой закон сохранения тепловой энергии

$$
\frac{\partial T}{\partial t}+\operatorname{div} \cdot \mathbf{q}=Q(\mathbf{r}, t)
$$

дополненный законом Фурье, связывающим тепловой поток q с градиентом температуры $T$ :

$$
\mathbf{q}+\sigma \operatorname{grad} \cdot T=0 ; \quad \sigma>0 .
$$

Вместе они образуют замкнутую систему уравнений параболического типа. Для нахождения однозначного решения этой системы необходимо задать начальные и граничные условия. Зададим их в виде

$$
\begin{aligned}
& T\left(\mathbf{r}, t_{0}\right)=\varphi(\mathbf{r}), \quad \mathbf{r} \in \Omega ;\left.T(\mathbf{r}, t)\right|_{\partial \Omega_{1}}=\left.\psi(\mathbf{r}, t)\right|_{\partial \Omega_{1}} ;\left.q_{n} \cdot(\mathbf{r}, t)\right|_{\partial \Omega_{3}}=\left.\theta(\mathbf{r}, t)\right|_{\partial \Omega_{3}} ; \\
& {\left.\left[T(\mathbf{r}, t)+\eta \cdot q_{n}(\mathbf{r}, t)\right]\right|_{\partial \Omega_{2}}=\left.\lambda(\mathbf{r}, t)\right|_{\partial \Omega_{2}} ; \partial \Omega_{1} \cup \partial \Omega_{2} \cup \partial \Omega_{3} \subseteq \partial \Omega}
\end{aligned}
$$

Возмущения решения, описываемого уравнениями (1),(2) распространяются с бесконечно большой скоростью, что противоречит современным физическим представлениям. По этой причине вместо соотношения (2) иногда используют его модификацию, устраняющую этот недостаток:

$$
\alpha \frac{\partial \mathbf{q}}{\partial t}+\operatorname{grad} \cdot T=-\sigma^{-1} \mathbf{q} .
$$

Здесь $\alpha$ - малый параметр, величина которого определяется из физических соображений в контексте конкретной решаемой задачи. В рамках данной работы мы не будем апеллировать к физическим представлениям и будем трактовать соотношение (4) как искусственное сингулярное возмущение закона Фурье - обобщенный закон Фурье. Наличие в (4) производной по вре- 
мени от потока требует задания его значения в начальный момент $t_{0}$. Определим его как

$$
\mathbf{q}\left(\mathbf{r}, t_{0}\right)=-\sigma \cdot \operatorname{grad}\left[T\left(\mathbf{r}, t_{0}\right)\right] .
$$

Покажем, что система уравнений (1),(4) является гиперболической. Будем называть систему уравнений первого порядка гиперболической, если для произвольного пространственного направления, задаваемого единичным вектором е, проекцию этой системы на заданное направление можно записать как

$$
\frac{\partial \psi}{\partial t}+\mathbf{A}_{\mathbf{e}} \frac{\partial \psi}{\partial \mathbf{e}}=\mathbf{D}
$$

где все собственные числа матрицы $\mathbf{A}_{\mathbf{e}}$ являются действительными и существует базис, в котором она имеет диагональный вид.

Следует отметить, что данное определение допускает возможность наличия у матрицы $\mathbf{A}_{\mathbf{e}}$ кратных собственных чисел, в то время как обычно требуется, чтобы все собственные числа были различными.

Приведем систему уравнений (1),(4) к виду (6). Пусть $\mathbf{e , k}, \boldsymbol{\tau}$ - левая тройка взаимно ортогональных единичных векторов, таких что

$$
(\mathbf{e} \cdot \boldsymbol{\tau})=(\boldsymbol{\tau} \cdot \mathbf{k})=(\mathbf{k} \cdot \mathbf{e})=0 ; \quad \mathbf{e} \times \mathbf{k}=\boldsymbol{\tau}, \mathbf{k} \times \boldsymbol{\tau}=\mathbf{e}, \boldsymbol{\tau} \times \mathbf{e}=\mathbf{k} .
$$

Введем обозначения: $q_{e}=\mathbf{q} \cdot \mathbf{e}, q_{k}=\mathbf{q} \cdot \mathbf{k}, q_{\tau}=\mathbf{q} \cdot \boldsymbol{\tau}$.

В новых обозначениях скалярное уравнение (1) примет вид

$$
\frac{\partial T}{\partial t}+\frac{\partial q_{e}}{\partial \mathbf{e}}=D_{T}(\mathbf{r}, t)=\left[Q(\mathbf{r}, t)-\frac{\partial q_{k}}{\partial \mathbf{k}}-\frac{\partial q_{\tau}}{\partial \boldsymbol{\tau}}\right]
$$

а обобщенный закон Фурье запишется как

$$
\begin{aligned}
& \alpha \frac{\partial q_{e}}{\partial t}+\frac{\partial T}{\partial \mathbf{e}}=D_{e}=-\sigma^{-1} \mathbf{q} ; \quad \alpha \frac{\partial q_{k}}{\partial t}=D_{k}=-\sigma^{-1} \mathbf{q}-\frac{\partial T}{\partial \mathbf{k}} ; \\
& \alpha \frac{\partial q_{\tau}}{\partial t}=D_{\tau}=-\sigma^{-1} \mathbf{q}-\frac{\partial T}{\partial \boldsymbol{\tau}} .
\end{aligned}
$$

Пусть $\boldsymbol{\psi}=\left(T, q_{e}, q_{k}, q_{\tau}\right)^{T}$, тогда систему уравнений $(7),(8)$ можно записать в матричном виде:

$$
\frac{\partial \psi}{\partial t}+\mathbf{A}_{e} \frac{\partial \psi}{\partial \mathbf{e}}=\mathbf{D} ; \quad \mathbf{D}=\left(D_{T}, D_{e}, D_{k}, D_{\tau}\right)^{T}
$$


где $\quad \mathbf{A}_{e}=\left(\begin{array}{cccc}0 & 1 & 0 & 0 \\ \alpha^{-1} & 0 & 0 & 0 \\ 0 & 0 & 0 & 0 \\ 0 & 0 & 0 & 0\end{array}\right) ; \quad\left\{\begin{array}{c}\left\|\mathbf{A}_{e}-\lambda \mathbf{E}\right\|=-\lambda^{4}+\lambda^{2} \alpha^{-1}=-\lambda^{2}\left(\lambda^{2}-\alpha^{-1}\right)=0, \\ \lambda_{1}=\alpha^{-1 / 2}, \quad \lambda_{2}=-\alpha^{-1 / 2}, \lambda_{3}=\lambda_{4}=0 .\end{array}\right.$

Найдем левые собственные векторы матрицы, отвечающие этим собственным числам:

$$
\mathbf{l}_{1}=\left(\alpha^{-1 / 2} 1,0,0\right) ; \quad \mathbf{l}_{2}=\left(-\alpha^{-1 / 2}, 1,0,0\right) ; \quad \mathbf{l}_{3}=(0,0,1,0) ; \quad \mathbf{l}_{4}=(0,0,0,1) .
$$

Умножая (6) на левые собственные векторы (9), приходим к характеристической форме уравнений вдоль направления $\mathbf{e}$ :

$$
\begin{aligned}
& \left(\frac{\partial q_{e}}{\partial t}+\alpha^{-1 / 2} \cdot \frac{\partial T}{\partial t}\right)+\alpha^{-1 / 2} \cdot\left(\frac{\partial q_{e}}{\partial \mathbf{e}}+\alpha^{-1 / 2} \cdot \frac{\partial T}{\partial \mathbf{e}}\right)=\alpha^{-1 / 2} \cdot D_{T}+D_{e} \\
& \left(\frac{\partial q_{e}}{\partial t}-\alpha^{-1 / 2} \cdot \frac{\partial T}{\partial t}\right)-\alpha^{-1 / 2} \cdot\left(\frac{\partial q_{e}}{\partial \mathbf{e}}-\alpha^{-1 / 2} \cdot \frac{\partial T}{\partial \mathbf{e}}\right)=-\alpha^{-1 / 2} \cdot D_{T}+D_{e} \\
& \frac{\partial q_{k}}{\partial t}=D_{k} ; \quad \frac{\partial q_{\tau}}{\partial t}=D_{\tau}
\end{aligned}
$$

Балансно-характеристический алгоритм. Рассмотрим случай двух пространственных измерений. Система уравнений (1),(4) в этом случае примет вид

$$
\frac{\partial T}{\partial t}+\frac{\partial q_{x}}{\partial x}+\frac{\partial q_{y}}{\partial y}=Q(x, y, t), \alpha \frac{\partial q_{x}}{\partial t}+\frac{\partial T}{\partial x}=-\sigma^{-1} q_{x} ; \alpha \frac{\partial q_{y}}{\partial t}+\frac{\partial T}{\partial y}=-\sigma^{-1} q_{y} .
$$

Малый параметр $\alpha$ при использовании сеточных численных методов естественно связать с характерными параметрами расчетной сетки, например, с шагом интегрирования по времени $(\alpha \sim \tau)$ или характерным размером расчетной ячейки $(\alpha \sim h)$. В этом случае гиперболические уравнения (1),(4) будут аппроксимировать исходную систему уравнений теплопроводности (1),(2) с первым порядком.

В области $G$ на плоскости $(x, y)$ рассмотрим расчетную сетку с ячейками в виде косоугольных четырехугольников (рис.1), согласованную с границами области. На этой сетке введем два сорта сеточных переменных т.н. «консервативные» переменные, относящиеся к центрам расчетных ячеек: $T_{c},\left(q_{x}\right)_{c},\left(q_{y}\right)_{c}$, и «потоковые» - определенные в серединах ребер: $T_{R}, T_{L}, T_{T}, T_{B} ;\left(q_{x}\right)_{R},\left(q_{x}\right)_{L},\left(q_{x}\right)_{T},\left(q_{x}\right)_{B} ;\left(q_{y}\right)_{R},\left(q_{y}\right)_{L},\left(q_{y}\right)_{T},\left(q_{y}\right)_{B}$. 
В начальный момент времени $t_{0}$ будем считать величины всех этих сеточных переменных известными. Балансно-характеристический алгоритм численного решения уравнения теплопроводности удобно записать в форме «предиктор - генератор потоков - корректор». На этапе «предиктор» вычисляются значения консервативных переменных на промежуточном временном слое. Затем на этапе «генератор потоков» находятся новые потоковые переменные и на этапе «корректор» вычисляются новые консервативные переменные.

Этап «предиктор»:

$$
\begin{gathered}
\frac{T_{c}^{n+1 / 2}-T_{c}^{n}}{\tau / 2}+\frac{1}{S} \cdot\left[\Delta y_{2,1} \cdot\left(q_{x}\right)_{R}^{n}+\Delta y_{3,2} \cdot\left(q_{x}\right)_{T}^{n}+\Delta y_{4,3} \cdot\left(q_{x}\right)_{L}^{n}+\Delta y_{1,4} \cdot\left(q_{x}\right)_{B}^{n}\right]- \\
-\frac{1}{S} \cdot\left[\Delta x_{2,1} \cdot\left(q_{y}\right)_{R}^{n}+\Delta x_{3,2} \cdot\left(q_{y}\right)_{T}^{n}+\Delta x_{4,3} \cdot\left(q_{y}\right)_{L}^{n}+\Delta x_{1,4} \cdot\left(q_{y}\right)_{B}^{n}\right]=Q_{c}^{n} ; \\
\alpha \cdot \frac{\left(q_{x}\right)_{c}^{n+1 / 2}-\left(q_{x}\right)_{c}^{n}}{\tau / 2}+\frac{1}{S} \cdot\left[\Delta y_{2,1} \cdot T_{R}^{n}+\Delta y_{3,2} \cdot T_{T}^{n}+\Delta y_{4,3} \cdot T_{L}^{n}+\Delta y_{1,4} \cdot T_{B}^{n}\right]=-\frac{1}{\sigma_{c}^{n}}\left(q_{x}\right)_{c}^{n+1 / 2} ; \\
\alpha \cdot \frac{\left(q_{y}\right)_{c}^{n+1 / 2}-\left(q_{y}\right)_{c}^{n}}{\tau / 2}-\frac{1}{S} \cdot\left[\Delta x_{2,1} \cdot T_{R}^{n}+\Delta x_{3,2} \cdot T_{T}^{n}+\Delta x_{4,3} \cdot T_{L}^{n}+\Delta x_{1,4} \cdot T_{B}^{n}\right]=-\frac{1}{\sigma_{c}^{n}}\left(q_{y}\right)_{c}^{n+1 / 2} .
\end{gathered}
$$

Здесь $S$ - площадь ячейки, остальные обозначения соответствуют рис.1.

Этап «генератор потоков».

На этапе «генератор потоков» используется характеристическая форма уравнений (10). В пределах одной пространственно-временной расчетной ячейки эти уравнения могут быть аппроксимированы следующим образом:

$$
\begin{aligned}
& \frac{\partial R_{\mathbf{e}}}{\partial t}+\lambda^{n+1 / 2} \cdot \frac{\partial R_{\mathbf{e}}}{\partial \mathbf{e}}=G_{R}^{n+1 / 2}=\left[\lambda^{n+1 / 2} \cdot D_{T}+D_{e}\right]_{c}^{n+1 / 2} \\
& \frac{\partial Q_{\mathbf{e}}}{\partial t}-\lambda^{n+1 / 2} \cdot \frac{\partial Q_{\mathbf{e}}}{\partial \mathbf{e}}=G_{Q}^{n+1 / 2}=\left[-\lambda^{n+1 / 2} \cdot D_{T}+D_{e}\right]_{c}^{n+1 / 2} \\
& \frac{\partial q_{k}}{\partial t}=\left(D_{k}\right)_{c}^{n+1 / 2}
\end{aligned}
$$

где

$$
R_{\mathbf{e}}=\mathbf{q} \cdot \mathbf{e}+\left\{\alpha^{-1 / 2}\right\}_{C}^{n+1 / 2} \cdot T ; \quad Q_{\mathbf{e}}=\mathbf{q} \cdot \mathbf{e}-\left\{\alpha^{-1 / 2}\right\}_{C}^{n+1 / 2} \cdot T
$$

- локальные инварианты Римана, а правые части $G_{R}^{n+1 / 2}, G_{Q}^{n+1 / 2},\left(D_{k}\right)_{c}^{n+1 / 2}$ считаются постоянными в пределах каждой ячейки. 
Для вычисления новых значений потоковых переменных на грани $C C$ (рис.2) в качестве вектора е выберем направление нормали к этой грани и вычислим значения инвариантов $\left(R_{\mathbf{e}}\right)_{L L}^{n},\left(R_{\mathbf{e}}\right)_{L C}^{n+1 / 2}$ и $\left(Q_{\mathbf{e}}\right)_{R R}^{n},\left(Q_{\mathbf{e}}\right)_{R C}^{n+1 / 2}$ :

$$
\begin{array}{ll}
\left(R_{\mathbf{e}}\right)_{L L}^{n}=\mathbf{q}_{L L}^{n} \cdot \mathbf{e}+a_{L} \cdot T_{L L}^{n} ; & \left(R_{\mathbf{e}}\right)_{L C}^{n+1 / 2}=\mathbf{q}_{L C}^{n+1 / 2} \cdot \mathbf{e}+a_{L} \cdot T_{L C}^{n+1 / 2} ; \\
\left(Q_{\mathbf{e}}\right)_{L L}^{n}=\mathbf{q}_{R R}^{n} \cdot \mathbf{e}-a_{R} \cdot T_{R R}^{n} ; & \left(Q_{\mathbf{e}}\right)_{R C}^{n+1 / 2}=\mathbf{q}_{R C}^{n+1 / 2} \cdot \mathbf{e}-a_{R} \cdot T_{R C}^{n+1 / 2},
\end{array}
$$

где $a_{L}=\left\{\alpha^{-1 / 2}\right\}_{L C}^{n+1 / 2} ; a_{R}=\left\{\alpha^{-1 / 2}\right\}_{R C}^{n+1 / 2}$.

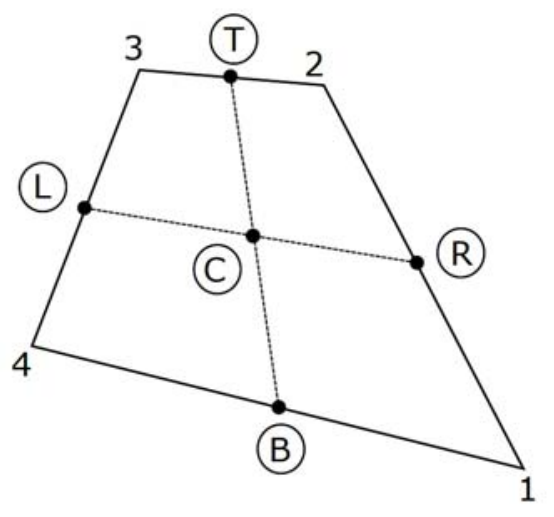

Puc.1.

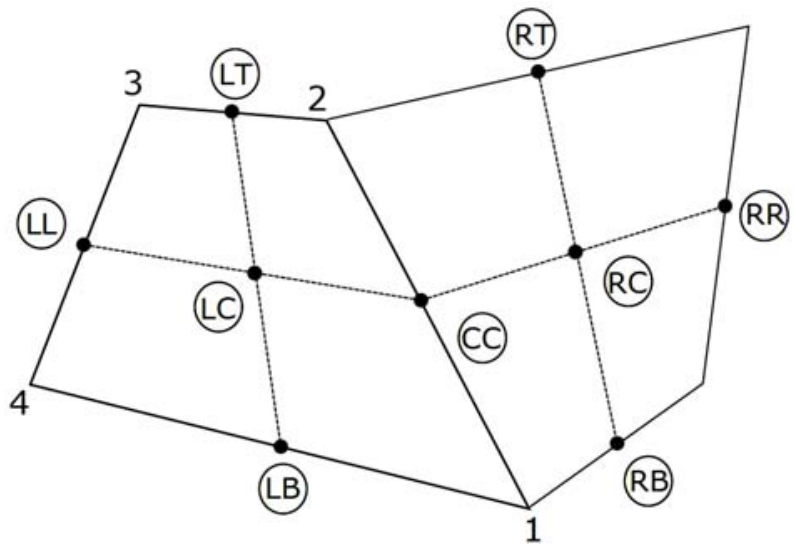

Рис.2.

Величины соответствующих инвариантов на новом временном слое вычислим линейной экстраполяцией:

$$
\left(R_{\mathbf{e}}\right)_{C C}^{n+1}=2\left(R_{\mathbf{e}}\right)_{L C}^{n+1 / 2}-\left(R_{\mathbf{e}}\right)_{L L}^{n} ;\left(Q_{\mathbf{e}}\right)_{C C}^{n+1}=2\left(Q_{\mathbf{e}}\right)_{R C}^{n+1 / 2}-\left(Q_{\mathbf{e}}\right)_{R R}^{n} .
$$

Для нахождения потоковых значений температуры и нормальной компоненты потока на новом временном слое получаем систему из двух уравнений:

$$
\mathbf{q}_{C C}^{n+1} \cdot \mathbf{e}+a_{L} \cdot T_{C C}^{n+1}=\left(R_{\mathbf{e}}\right)_{C C}^{n+1} ; \quad \mathbf{q}_{C C}^{n+1} \cdot \mathbf{e}-a_{R} \cdot T_{C C}^{n+1}=\left(Q_{\mathbf{e}}\right)_{C C}^{n+1} ;
$$

откуда находим:

$$
T_{C C}^{n+1}=\frac{\left(R_{\mathbf{e}}\right)_{C C}^{n+1}-\left(Q_{\mathbf{e}}\right)_{C C}^{n+1}}{a_{L}+a_{R}} ; \quad \mathbf{q}_{C C}^{n+1} \cdot \mathbf{e}=\frac{\left(R_{\mathbf{e}}\right)_{C C}^{n+1} \cdot a_{R}+\left(Q_{\mathbf{e}}\right)_{C C}^{n+1} \cdot a_{L}}{a_{L}+a_{R}} .
$$

Для вычисления касательной компоненты потока $\mathbf{q}_{C C}^{n+1} \cdot \mathbf{k}$ воспользуемся третьим уравнением системы (11). Правые части, постоянные для каждой ячейки, вычислим по известным значениям на промежуточном шаге по времени:

$$
\left(D_{k}\right)_{L C}^{n+1 / 2}=\frac{\left(q_{k}\right)_{L C}^{n+1 / 2}-\left(q_{k}\right)_{L C}^{n}}{\tau / 2} ; \quad\left(D_{k}\right)_{R C}^{n+1 / 2}=\frac{\left(q_{k}\right)_{R C}^{n+1 / 2}-\left(q_{k}\right)_{R C}^{n}}{\tau / 2} .
$$


Затем вычисляем эту компоненту потока, относящуюся к центру ячеек, на новом временном слое:

$$
\frac{\left(q_{k}\right)_{L C}^{n+1}-\left(q_{k}\right)_{L C}^{n}}{\tau}=\left(D_{k}\right)_{L C}^{n+1 / 2} ; \quad \frac{\left(q_{k}\right)_{R C}^{n+1}-\left(q_{k}\right)_{R C}^{n}}{\tau}=\left(D_{k}\right)_{R C}^{n+1 / 2},
$$

и интерполируем эти результаты на середину ребра

$$
\left(q_{k}\right)_{C C}^{n+1}=\frac{\Delta l_{L} \cdot\left(q_{k}\right)_{R C}^{n+1}+\Delta l_{R} \cdot\left(q_{k}\right)_{L C}^{n+1}}{\Delta l_{L}+\Delta l_{R}} .
$$

Потоковые переменные на других ребрах вычисляются аналогично.

Этап «корректор»

$$
\begin{aligned}
& \frac{T_{c}^{n+1}-T_{c}^{n}}{\tau}+\frac{1}{S} \cdot\left[\Delta y_{2,1} \cdot\left(q_{x}\right)_{R}^{n+1}+\Delta y_{3,2} \cdot\left(q_{x}\right)_{T}^{n+1}+\Delta y_{4,3} \cdot\left(q_{x}\right)_{L}^{n+1}+\Delta y_{1,4} \cdot\left(q_{x}\right)_{B}^{n+1}\right]- \\
& -\frac{1}{S} \cdot\left[\Delta x_{2,1} \cdot\left(q_{y}\right)_{R}^{n+1}+\Delta x_{3,2} \cdot\left(q_{y}\right)_{T}^{n+1}+\Delta x_{4,3} \cdot\left(q_{y}\right)_{L}^{n+1}+\Delta x_{1,4} \cdot\left(q_{y}\right)_{B}^{n+1}\right]=Q_{c}^{n+1 / 2} \\
& \alpha \cdot \frac{\left(q_{x}\right)_{c}^{n+1}-\left(q_{x}\right)_{c}^{n}}{\tau}+\frac{1}{S} \cdot\left[\Delta y_{2,1} \cdot T_{R}^{n+1}+\Delta y_{3,2} \cdot T_{T}^{n+1}+\Delta y_{4,3} \cdot T_{L}^{n+1}+\Delta y_{1,4} \cdot T_{B}^{n+1}\right]= \\
& =-\frac{1}{\sigma_{c}^{n}}\left(q_{x}\right)_{c}^{n+1} ; \\
& \alpha \cdot \frac{\left(q_{y}\right)_{c}^{n+1}-\left(q_{y}\right)_{c}^{n}}{\tau}-\frac{1}{S} \cdot\left[\Delta x_{2,1} \cdot T_{R}^{n+1}+\Delta x_{3,2} \cdot T_{T}^{n+1}+\Delta x_{4,3} \cdot T_{L}^{n+1}+\Delta x_{1,4} \cdot T_{B}^{n+1}\right]= \\
& =-\frac{1}{\sigma_{c}^{n}}\left(q_{y}\right)_{c}^{n+1} \cdot
\end{aligned}
$$

Порядок аппроксимации представленного алгоритма зависит от выбора параметра $\alpha$. Если $\alpha \simeq \tau$, то схема будет иметь первый порядок аппроксимации по времени и второй (на «гладких» сетках) по пространственным переменным. При $\alpha \simeq \Delta l-$ первый порядок как по времени, так и по пространству.

Граничные условия. Если правое ребро (рис.1) принадлежит границе области, то на нем задается граничное условие первого, второго или третьего рода. На правое ребро со стороны ячейки приходит локальный инвариант $\mathbf{q}_{R}^{n+1} \cdot \mathbf{e}+a_{c} \cdot T_{R}^{n+1}=\left(R_{\mathbf{e}}\right)_{R}^{n+1} ; a_{c}=\left\{\alpha^{-1 / 2}\right\}_{C}^{n+1 / 2}$, значение которого известно. Если на ребре задано значение температуры $T_{R}^{n+1}$, то по значению локаль- 
ного инварианта $\left(R_{\mathbf{e}}\right)_{R}^{n+1}$ однозначно находится величина нормального потока $\mathbf{q}_{R}^{n+1} \cdot \mathbf{e}$. Если задан поток, то однозначно вычисляется температура. В случае граничного условия третьего рода получаем систему уравнений:

$$
\begin{aligned}
& \mathbf{q}_{R}^{n+1} \cdot \mathbf{e}+a_{c} \cdot T_{R}^{n+1}=\left(R_{\mathbf{e}}\right)_{R}^{n+1} \\
& \eta \cdot \mathbf{q}_{R}^{n+1} \cdot \mathbf{e}+T_{R}^{n+1}=\lambda_{R}^{n+1} .
\end{aligned}
$$

При условии $\eta \cdot a_{c} \neq 1$ эта система однозначно разрешима. Ввиду довольно широкого произвола в выборе параметра $\alpha$ последнее всегда может быть выполнено.

Граничные условия на других ребрах реализуются аналогично.

Вычисление шага интегрирования по времени. Для явных балансно характеристических разностных схем гиперболического типа область устойчивости определяется размерами расчетных ячеек и скоростью распространения сигнала. В нашем случае это приводит к ограничению:

$$
\frac{\tau}{\Delta l \cdot \sqrt{\alpha}}<1
$$

Если $\alpha=\tau$, то асимптотическое поведение области устойчивости будет таким же, как и у явной схемы для уравнения теплопроводности: $\tau / \Delta l^{2} \simeq 1$. При $\alpha=\Delta l$ асимптотика изменится: $\tau / \Delta l^{3 / 2} \simeq 1$. Таким образом, область устойчивости может быть расширена за счет уменьшения порядка аппроксимации. Если положить $\alpha=\Delta l^{\delta}, \delta=1-\varepsilon, \varepsilon \in[0,1]$, то область устойчивости можно расширить еще больше: $\tau / \Delta l^{(3-\varepsilon) / 2} \simeq 1$. При совместном решении системы уравнений газовой динамики и уравнения теплопроводности это позволяет использовать явные алгоритмы на более подробных расчетных сетках. Следует отметить, что от коэффициента теплопроводности область устойчивости балансно-характеристической схемы не зависит.

Примеры численных расчетов. Решение параболических уравнений по балансно-характеристическим разностным схемам имеет ряд особенностей. Рассмотрим их на примере одномерной задачи теплопроводности с дельтаобразным источником.

В случае $\alpha=\tau$ область устойчивости БХРС формально оказывается в два раза больше, однако сходимость к аналитическому решению наблюдается только при $C F L \leq 0.5$. На рис.За приведены результаты сравнения численного и аналитического решения задачи на безразмерный момент време- 
ни $t=0.001$. В качестве характерных выбраны следующие параметры: Характерный пространственный масштаб $L=1$, характерный временной масштаб $t^{*}=2 \cdot \sigma / L^{2}$. Чрезвычайно важным моментом при этом является то обстоятельство, что в БХРС алгоритме используется коррекция потоков в соответствии с принципом максимума. Если эту коррекцию отключить, то сходимости численного решения к аналитическому не будет, что иллюстрирует рис.3б. Рис.3в иллюстрирует потерю монотонности решения при числах Куранта-Фридрихса-Леви, превышающих 0.5 .

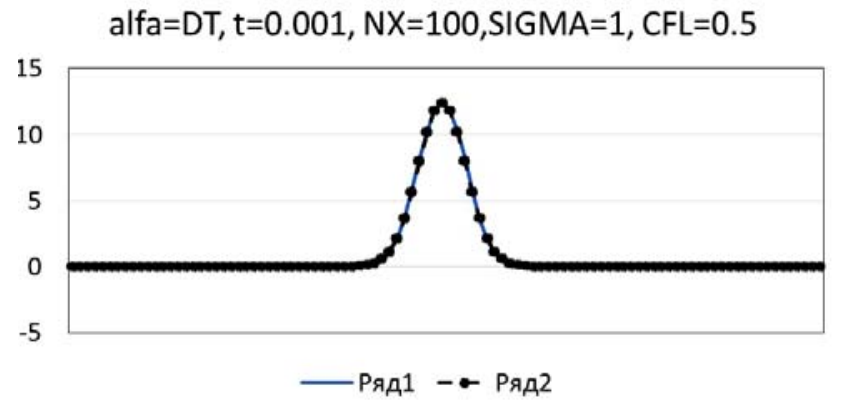

a

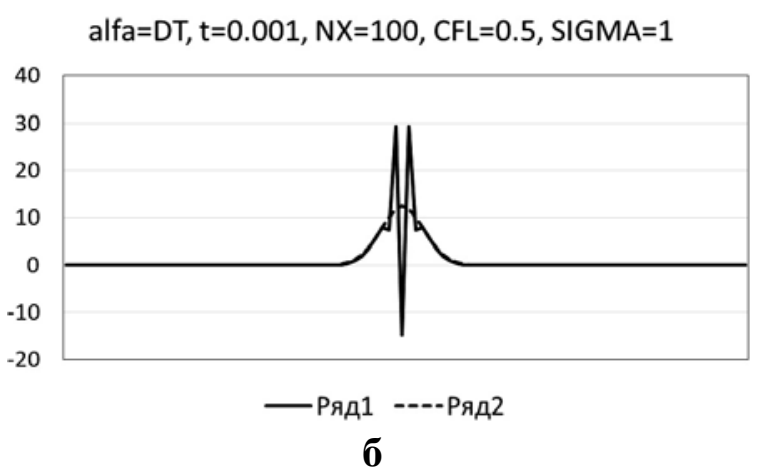

б

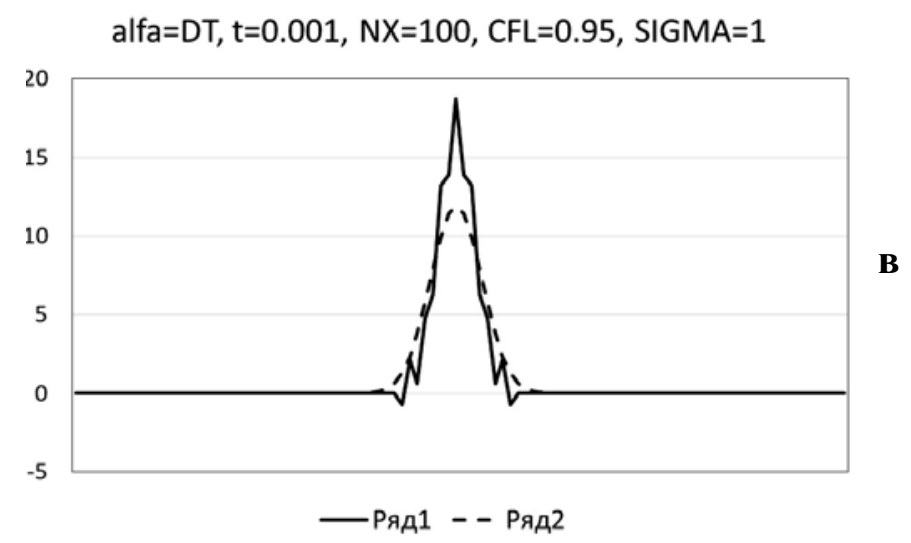

Рис.3a,б,в.

Как было отмечено ранее, область устойчивости БХРС для параболических уравнений не зависит от коэффициента теплопроводности. Если его увеличить, например, в 10 раз, то это будет соответствовать фактическому увеличению во столько же раз и теплового числа CFL. Однако это не может не сказаться на качестве расчетов. На рис. 4 приведено сравнение численного и аналитического решения на один и тот же момент времени на сгущающихся сетках. На рис.4а хорошо видны две разбегающиеся тепловые волны, являющиеся отголоском насильственной гиперболизации. На рис.4б, в показана сходимость «гиперболизованного» решения к фундаментальному решению уравнения теплопроводности. Относительно быстрый характер сходимости обусловлен тем, что БХРС при $\alpha=\tau$ имеет второй порядок аппроксимации по пространству. 


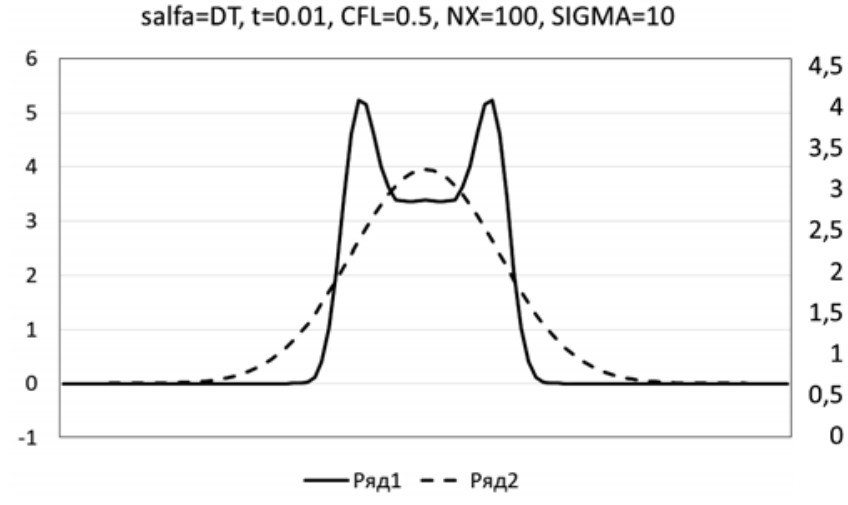

$\mathbf{a}$

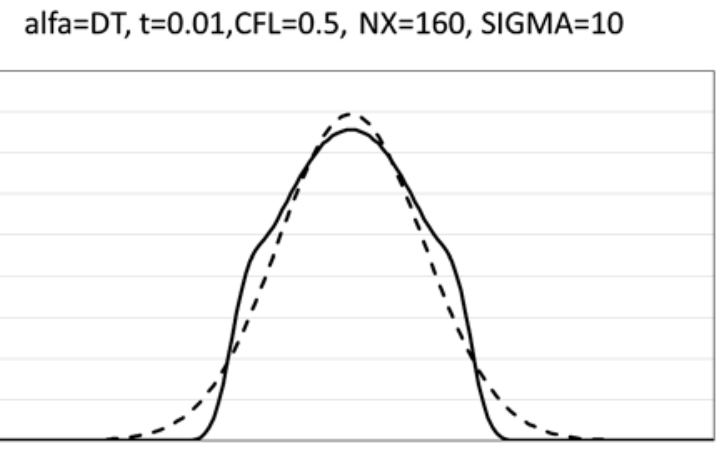

—Ряд1 - - Ряд2

б

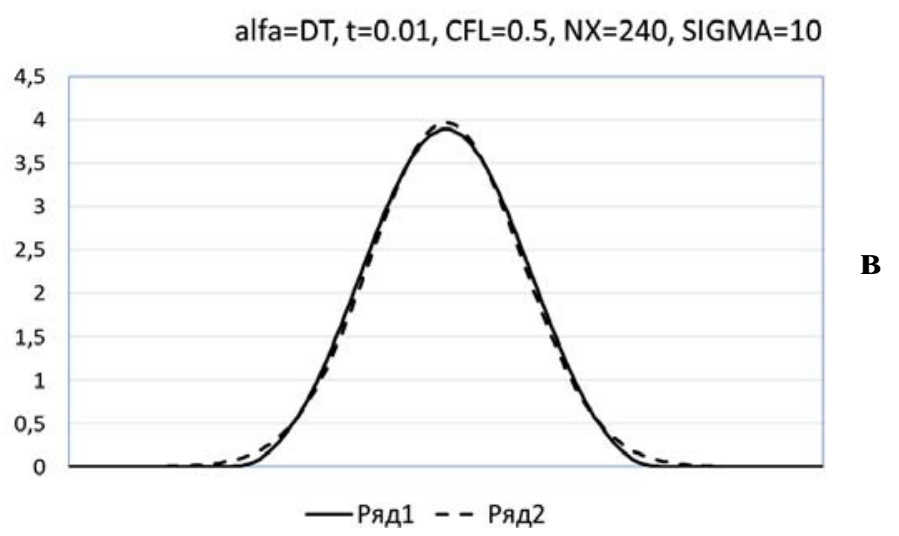

Puc.4a, б,в.

Следует отметить, что расчеты, представленные на рис.4, проводились при $C F L=0.5$, что в данном случае соответствует тепловому $C F L=5$.

При $\alpha=\Delta x$ порядок аппроксимации по пространству уменьшается до первого, что проявляется в существенно более медленной сходимости к фундаментальному решению на тот же момент времени. Этот факт иллюстрируется на рис.5. Следует обратить внимание на то, что эффективное тепловое число CFL составляет несколько десятков и растет со сгущением сетки. Приведенные расчеты подтверждают качественные оценки, приведенные в [4].

Далее рассмотрим пример расчета уравнения теплопроводности с дельтообразным источником на плоскости с криволинейной сеткой. На рис.6 приведена криволинейная расчетная сетка в квадратной области $\Omega=$ $=[0,1] \times[0,1]$, полученная из ортогональной сетки путем поворота центральных узлов сетки на 90 градусов против часовой стрелки. Расчет проводился при $\alpha=\tau$ с числом Куранта (тепловым) $\mathrm{CFL}=5$. Результаты расчета приведены на рис. 6 и 7. Распределение температуры в момент времени $t=0.015$ показано на рис.6. На рис.7 приведены зависимости температуры от расстояния до центра области (радиуса) в различные моменты времени $(t=$ $=0.005, t=0.01$ и $t=0.025)$. Результаты расчета сравниваются с аналитическим решением - фундаментальным решением уравнения теплопроводности. Видно, что на криволинейной расчетной сетке решение остается изотропным и совпадает с аналитическим решением. 


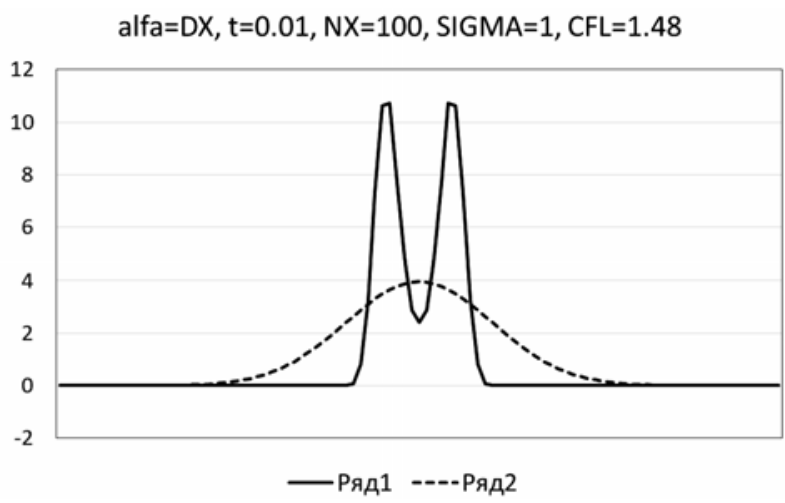

$\mathbf{a}$

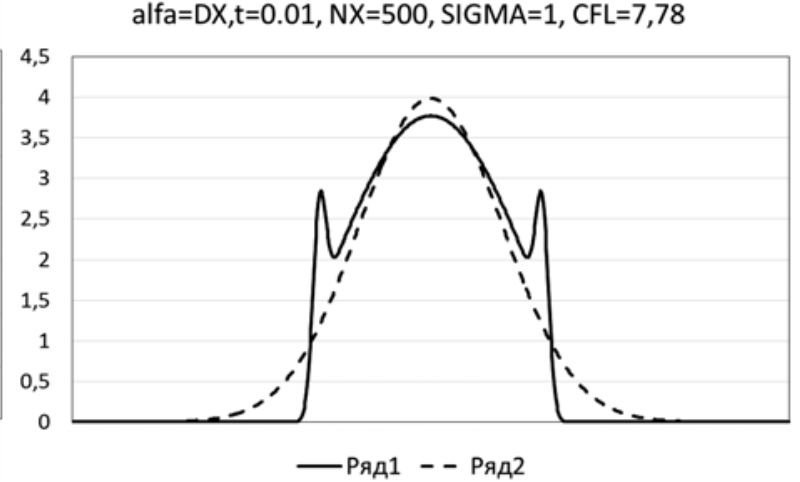

б

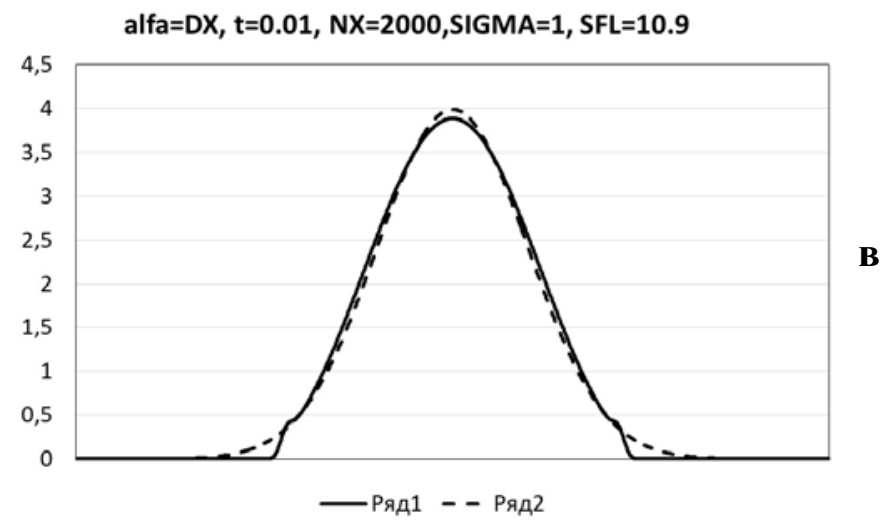

Pис.5a,б,в.

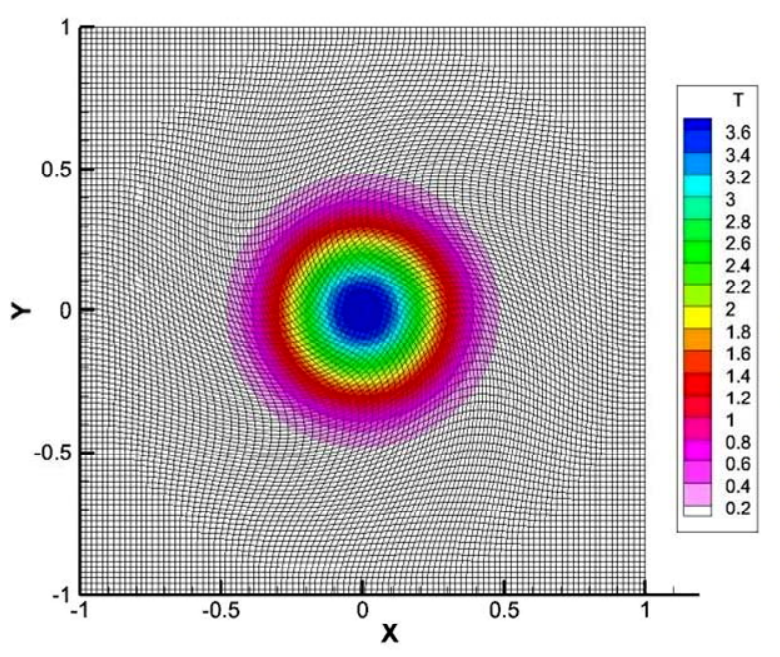

Рис.6. Криволинейная сетка. Температура в момент времени $\mathrm{t}=0.015 \mathrm{c}$.

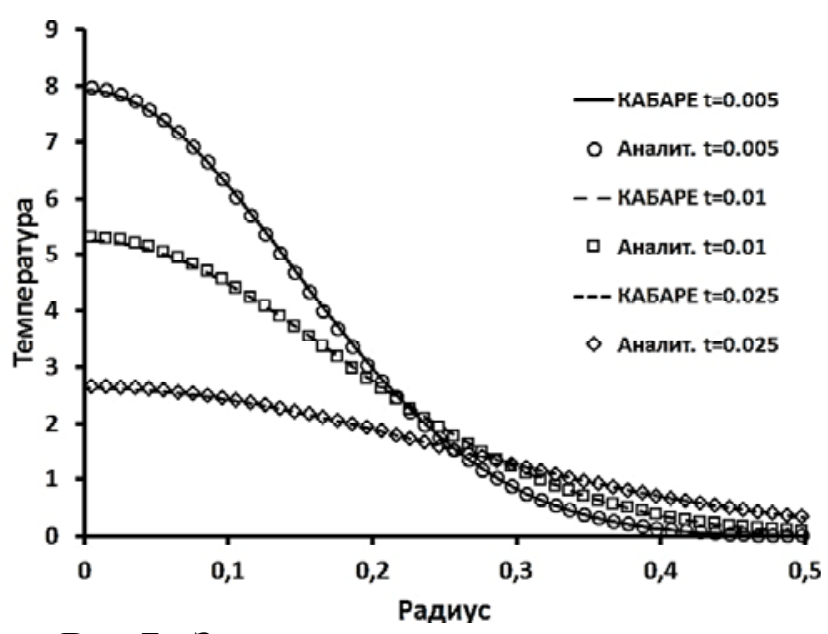

Рис.7. Зависимость температуры от расстояния до центра области в различные моменты времени.

Заключение. Предложен новый численный алгоритм для решений параболических уравнений по балансно-характеристическим разностным схемам, объединяющим достоинства консервативных и характеристических разностных схем [5]. Основным достоинством нового алгоритма является то, что он является явным и реализуется на максимально компактном вы- 
числительном шаблоне в одну пространственно-временную расчетную ячейку. Он идеально масштабируется и оказывается чрезвычайно экономичным в программных комплексах, реализующих схему КАБАРЕ [6], поскольку использует уже существующую там структуру данных.

\section{СПИСОК ЛИТЕРАТУРЫ}

1. В.М. Головизнин, И.А. Короткин, С.А. Финогенов. Моделирование турбулентной естественной конвекции в замкнутых вытянутых по высоте областях // Вычислительная механика сплошных сред, 2016, т.9, № 3, с.253-263;

V.M. Goloviznin, I.A. Korotkin, S.A. Finogenov. Modelirovanie turbulentnoi estestvennoi konvektsii v zamknutykh vytianutykh po vysote oblastiakh // Vychislitelnaia mekhanika sploshnykh sred, 2016, t.9, № 3, s.253-263.

2. В.Ю. Глотов, В.М. Головизнин, К.М. Сергеенко. LES-моделирование турбулентного теплообмена при течении свинцового теплоносителя в круглой трубе при различных числах Рейнольдса // Математическое моделирование, 2018, т.30, №7, с.46-53;

V.Iu. Glotov, V.M. Goloviznin, K.M. Sergeenko. LES-modelirovanie turbulentnogo teploobmena pri techenii svintsovogo teplonositelia $\mathrm{v}$ krugloi trube pri razlichnykh chislakh Reinoldsa // Matematicheskoe modelirovanie, 2018, t.30, №7, s.46-53.

3. Д.Г. Асфандияров, В.М. Головизнин, С.А. Финогенов. Беспараметрический метод расчета турбулентного течения в плоском канале в широком диапазоне чисел Рейнольдса // ЖВМиМФ, 2015, т.55, № 9, с.1545-1558;

D.G. Asfandiyarov, V.M. Goloviznin, S.A. Finogenov. Parameter-free method for computing the turbulent flow in a plane channel in a wide range of Reynolds numbers // Computational Mathematics and Mathematical Physics, 2015, v.55:9, p.1515-1526.

4. С.И. Репин, Б.Н. Четверушкин. Оценка разности приближенных решений задач Коши для параболического уравнения и гиперболического уравнения с малым параметром // ДАН, 2013, т.451, 3, с.255-258;

S.I. Repin, B.N. Chetverushkin. Estimates of the difference between approximate solutions of the cauchy problems for the parabolic diffusion equation and a hyperbolic equation with a small parameter // Doklady Mathematics, 2013, v.88, №1, p.417-420.

5. В.М. Головизнин, Б.Н. Четверушкин. Алгоритмы нового поколения в вычислительной гидродинамике // ЖВМиМФ, 2018, т.58, №8, с.20-29;

V.M. Goloviznin, B.N. Chetverushkin. New generation algorithms for computational fluid dynamics // Comput. Mathem. and Mathem. Physics, 2018, v.58, Issue 8, p.1217-1225.

6. В.М. Головизнин, М.А. Зайцев, С.А. Карабасов, И.А. Короткин. Новые алгоритмы вычислительной гидродинамики для многопроцессорных вычислительных систем // Издательство Московского университета. - М.: 2013, 467 с.;

V.M. Goloviznin, M.A. Zaitsev, S.A. Karabasov, I.A. Korotkin. Novye algoritmy vychislitelnoi gidrodinamiki dlia mnogoprotsessornykh vychislitelnykh sistem // Izdatelstvo Moskovskogo universiteta. - M.: 2013, 467 s.

Поступила в редакцию 09.09.2019

После доработки 09.09.2019

Принята к публикации 21.10.2019 